\title{
Magnetization of graphane by dehydrogenation
}

H. Şahin, C. Ataca, and S. Ciraci

Citation: Appl. Phys. Lett. 95, 222510 (2009);

View online: https://doi.org/10.1063/1.3268792

View Table of Contents: http://aip.scitation.org/toc/apl/95/22

Published by the American Institute of Physics

\section{Articles you may be interested in}

Electronic structures of graphane with vacancies and graphene adsorbed with fluorine atoms AIP Advances 2, 012173 (2012); 10.1063/1.3696883

The response of mechanical and electronic properties of graphane to the elastic strain Applied Physics Letters 96, 091912 (2010); 10.1063/1.3353968

Spintronic properties of zigzag-edged triangular graphene flakes Journal of Applied Physics 108, 074301 (2010); 10.1063/1.3489919

Understanding adsorption of hydrogen atoms on graphene

The Journal of Chemical Physics 130, 054704 (2009); 10.1063/1.3072333

Silicene: Recent theoretical advances

Applied Physics Reviews 3, 040802 (2016); 10.1063/1.4944631

Perfect spin-filter and spin-valve in carbon atomic chains

Applied Physics Letters 96, 042104 (2010); 10.1063/1.3299264

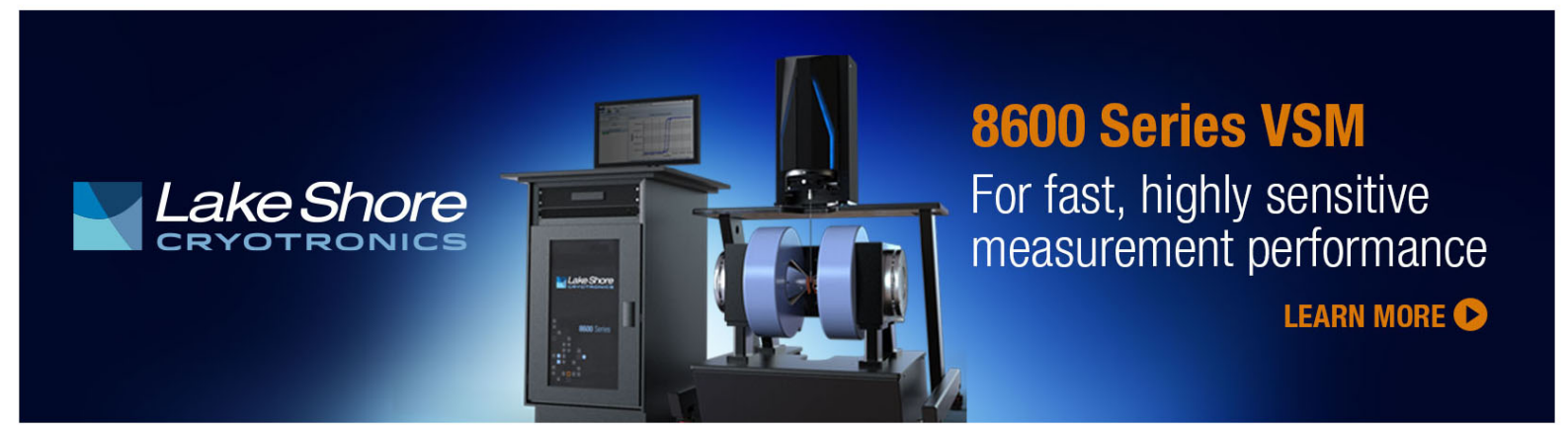




\title{
Magnetization of graphane by dehydrogenation
}

\author{
H. Şahin, ${ }^{1, a)}$ C. Ataca, ${ }^{1,2}$ and S. Ciraci ${ }^{1,2, b)}$ \\ ${ }^{1}$ UNAM-Institute of Materials Science and Nanotechnology, Bilkent University, 06800 Ankara, Turkey \\ ${ }^{2}$ Department of Physics, Bilkent University, 06800 Ankara, Turkey
}

(Received 21 October 2009; accepted 4 November 2009; published online 3 December 2009)

\begin{abstract}
Using first principles calculations, we show that each hydrogen vacancy created at graphane surface results in a local unpaired spin. For domains of hydrogen vacancies the situation is, however, complex and depends on the size and geometry of domains, as well as whether the domains are single or double sided. In single-sided domains, hydrogen atoms at the other side are relocated to pair the spins of adjacent carbon atoms by forming $\pi$-bonds. Owing to the different characters of exchange coupling in different ranges and interplay between unpaired spin and the binding geometry of hydrogen, vacancy domains can attain sizable net magnetic moments. (C) 2009 American Institute of Physics. [doi:10.1063/1.3268792]
\end{abstract}

Graphene, ${ }^{1}$ a truly two-dimensional (2D) crystal of honeycomb structure, has sparked considerable interest not only because of its charge carriers behaving like massless Dirac fermions, ${ }^{2-4}$ but also the unusual magnetic properties displayed by its flakes and nanoribbons. ${ }^{5-10}$ In addition to numerous experimental and theoretical studies on the physical properties of graphene, efforts have been also devoted to synthesize various types of derivatives of graphene. More recently, a 2D hydrocarbon material in the family of honeycomb structure, namely graphane is synthesized. ${ }^{11}$ Interesting properties such as reversible hydrogenationdehydrogenation with changing temperature, ${ }^{11}$ the electronic structure with a wide band gap ${ }^{12,13}$ have been revealed soon after its synthesis. In this letter, we reveal that graphane can be magnetized by dehydrogenation of domains on its surfaces. Large magnetic moments can be attained in a small domain on the graphane sheet, depending on whether the defect region is one sided or two sided. Our predictions are obtained from the state-of-the-art first-principles planewave calculations within the local density approximation (LDA). ${ }^{14,15}$ The structures are treated using periodically repeating $(11 \times 11 \times 1)$ supercells. Spin-polarized calculations include noncollinear magnetism with spin orbit interaction. Details of our method can be found in Refs. 16 and 17.

Graphane, in its chair conformation as illustrated in Fig. 1(a), is derived by the adsorption of a single hydrogen atom to each carbon atom alternating between the top $(A)$ and bottom $(B)$ side in the honeycomb structure. A charge of 0.1 electrons is transferred from $\mathrm{H}$ to $\mathrm{C}$ leaving behind positively charged $\mathrm{H}$ atoms on both sides of a double layer of negatively charged ( -0.1 electrons) $\mathrm{C}$ atoms. Graphane having a 2D quadruple structure has the work function $\Phi=4.97 \mathrm{eV}$, which is $\sim 0.2 \mathrm{eV}$ larger than that of graphene. In contrast to semimetallic graphene, graphane is a semiconductor with a wide direct band gap of $3.42 \mathrm{eV}$ calculated by LDA but corrected to be $5.97 \mathrm{eV}$ with $\mathrm{GW}_{0}$ self-energy method, as shown in Fig. 1(b). Doubly degenerate states at the $\Gamma$-point at the top of the valence band are derived from $2 p_{x^{-}}$and $2 p_{y}$-orbitals of carbon atoms. The edge of the conduction band is composed mainly from C- $p_{z}$ orbitals. Calculated pho-

\footnotetext{
${ }^{a)}$ Electronic mail: shasan@ bilkent.edu.tr.

${ }^{b)}$ Electronic mail: ciraci@fen.bilkent.edu.tr.
}

non bands all having positive frequencies confirm the stability of 2D graphane. High frequency vibration modes associated with $\mathrm{C}-\mathrm{H}$ bonds are well separated from the rest of the spectrum in Fig. 1(c).

The creation of a single H-vacancy at the hydrogen covered surfaces gives rise to the spin polarization in the nonmagnetic perfect graphane. Desorption of a single $\mathrm{H}$ atom from graphane is an endothermic reaction with $4.79 \mathrm{eV}$ en-
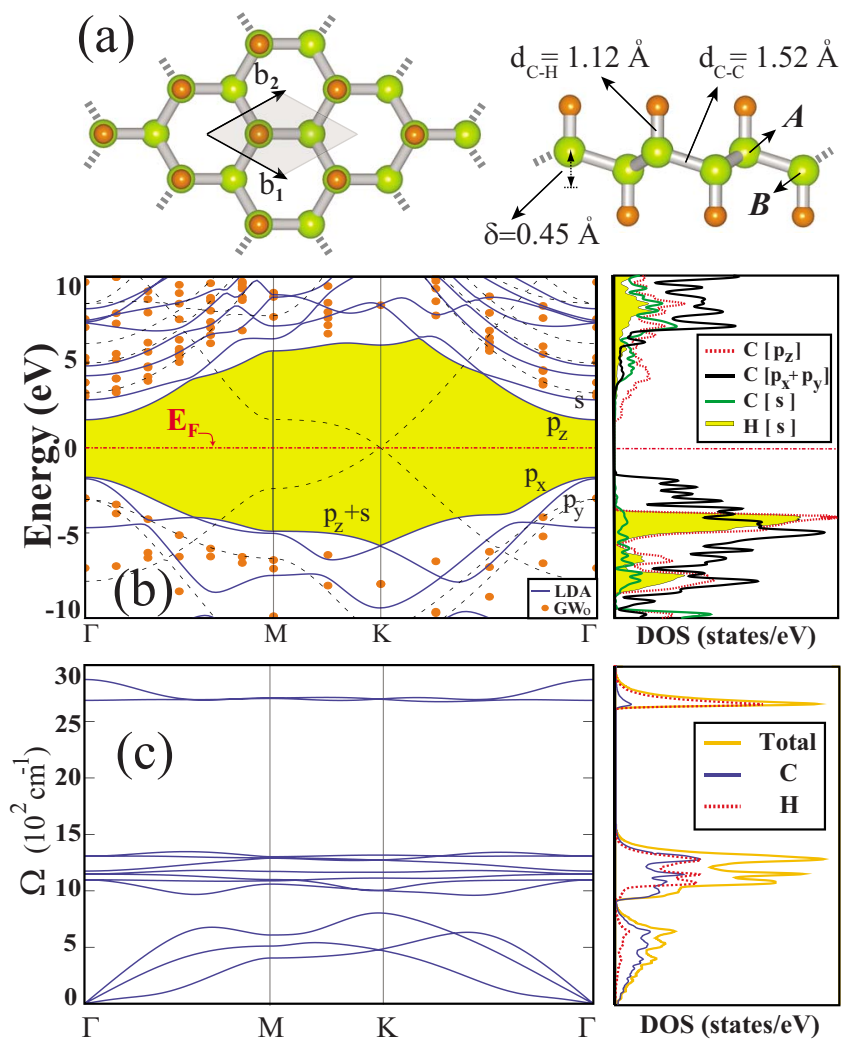

FIG. 1. (Color online) (a) Top and side views of atomic structure showing of graphane primitive unit cell with Bravais lattice vectors $\mathbf{b}_{1}$ and $\mathbf{b}_{2}$ and buckling of alternating carbon atoms, $\mathrm{A}$ and $\mathrm{B}$, in honeycomb structure $\delta$, bond lengths $d_{\mathrm{C}-\mathrm{C}}$ and $d_{\mathrm{C}-\mathrm{H}}$ optimized using LDA. Large green (light) and small orange (dark) balls indicate $\mathrm{C}$ and $\mathrm{H}$ atoms, respectively. (b) Energy band structure is calculated by using LDA and corrected using $\mathrm{GW}_{0}$ (shown by blue lines and orange dots). For graphene, linear band crossing at Dirac point is shown by dashed gray lines. (c) Calculated phonon bands and density of states DOS projected to $\mathrm{C}$ and $\mathrm{H}$ atoms. 

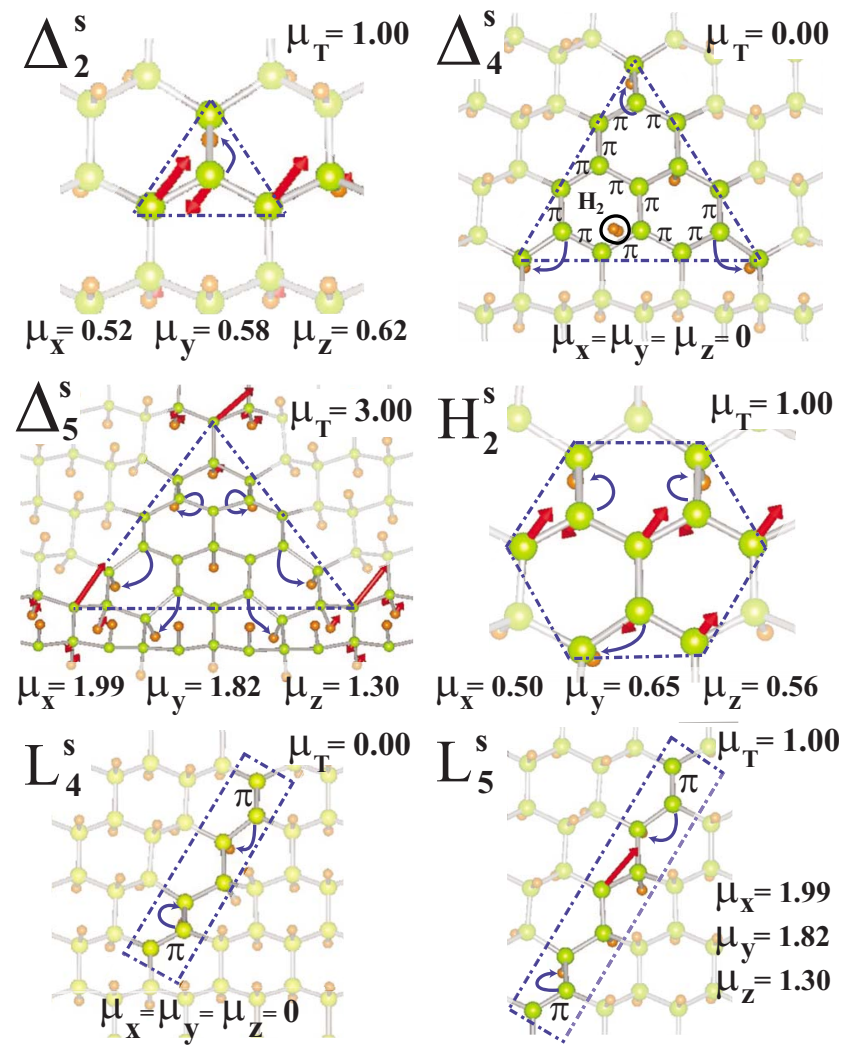

FIG. 2. (Color online) Calculated magnetic state of various domains of single-sided $\mathrm{H}$-vacancies, where all $\mathrm{H}$ atoms attached to $\mathrm{C}$ atoms from upper side in the unshaded region (delineated by dashed-dotted lines) including edges, are removed. The triangles are specified by $\Delta_{n}^{s}$ with $n$ indicating the maximum number of $\mathrm{C}$ atoms at one edge and $s$ signifies the single-sided dehydrogenation. Similar symbols are used also for hexagonal, $H_{2}^{s}$ and lane $L_{n}^{s}(n=4,5)$ domains. Total magnetic moment $\mu_{T}$ and its components $\mu_{x}, \mu_{y}$, and $\mu_{z}$ are given in units of the Bohr magneton $\mu_{B}$. Magnetic moments on $\mathrm{C}$ atoms are shown by red (black) arrows. Relocations of $\mathrm{H}$ atoms at the other side of graphane are shown by curly arrows. For the sake of clarity $\pi$-bonds formed after the relocation of bottom $\mathrm{H}$ atoms are indicated only for $\Delta_{4}^{s}, L_{4}^{s}$, and $L_{5}^{S}$ structures.

ergy. Various techniques, such as laser beam resonating with surface-hydrogen bond, ${ }^{18}$ stripping with ionic vapor, ${ }^{99}$ and scission of $\mathrm{C}-\mathrm{H}$ bonds with subnanometer Pt clusters, ${ }^{20}$ can be used to create $\mathrm{H}$-vacancy(ies). Upon desorption of a single hydrogen atom, local bonding through $s p^{3}$ hybrid orbital is retransformed into planar $s p^{2}$ and perpendicular $p_{z}$ $(\pi)$ orbitals. At the vacancy site one unpaired electron accommodated by the dangling $p_{z}$ orbital contributes to the magnetization by one $\mu_{B}$ (i.e., Bohr magneton). The exchange interaction between two $\mathrm{H}$-vacancies calculated in a $(11 \times 11 \times 1)$ supercell is found to be nonmagnetic for the first and second nearest neighbor distances due to spin pairings. Since the $\pi-\pi$ interaction vanishes for farther distances, antiferromagnetic (AFM) state between two H-vacancies for the third and fourth nearest neighbor distance is energetically favorable. The occurrence of long range spin interactions in carbon based structures was explained before by the superexchange $^{21}$ and magnetic tail interaction. ${ }^{22}$

As for the islands of $\mathrm{H}$-vacancies at the single (top) side of graphane, we consider various geometrical domains, where $\mathrm{H}$ atoms at their edges and inside are removed as seen in Fig. 2. For a triangular domain specified as $\Delta_{2}^{s}$ at the top side, $\mathrm{H}$ atoms attached to three carbon atoms located at each edge are removed. Hydrogen atom which is normally ad- sorbed on the central $\mathrm{C}$ atom at the bottom side moves to the corner. Under these circumstances, spins of three hydrogenfree $\mathrm{C}$ atoms are antiferromagnetically ordered to yield a net magnetic moment of $1 \mu_{B}$. Noncollinear calculations with spin-orbit interaction fix the directions of spins, which are tilted relative to the normal to the graphane plane. For $\Delta_{4}^{s}$, a triangular domain has ten $\mathrm{H}$ atoms removed from the top side of graphane. While part of six $\mathrm{H}$ atoms are attached to carbon atoms from bottom are relocated, remaining two $\mathrm{H}$ atoms are released by forming $\mathrm{H}_{2}$ molecule. At the end spins are paired and the net magnetic moment of the domain becomes vanished. Generally, for a small single-sided domain, $\mu_{T}=0$ if $N_{t}$, the total number of $\mathrm{H}$ atoms stripped, is an even number so that adjacent $\pi$-orbitals form spin paired $\pi$-bonds. In this case, $\mathrm{H}$ atoms below the domain are relocated (without facing any energy barrier) to pair adjacent $\pi$-orbitals to form maximum number of $\pi$-bonds. At the end, a large buckled regions inside the domain tends to be flattened and reconstructed to make nonmagnetic graphenelike planar structure. In the case of $\Delta_{5}^{s}$, while spins are paired through the formation of $\pi$-bonding between two adjacent $\mathrm{C}$ atoms following the relocation $\mathrm{H}$ atoms at the bottom side, the unpaired spins at the corner atoms are aligned in the same direction to yield a net magnetic moment of $\mu_{T}=3 \mu_{B}$. The tendency to pair the spins of adjacent $\mathrm{C}$ atoms to form $\pi$-bonds are seen better in lane domains. Let us consider $L_{4}^{s}$ and $L_{5}^{s}$ in Fig. 2. Because of relocation of $\mathrm{H}$ atoms at the bottom side, two pairs of nearest neighbor $\mathrm{C}$ atoms form
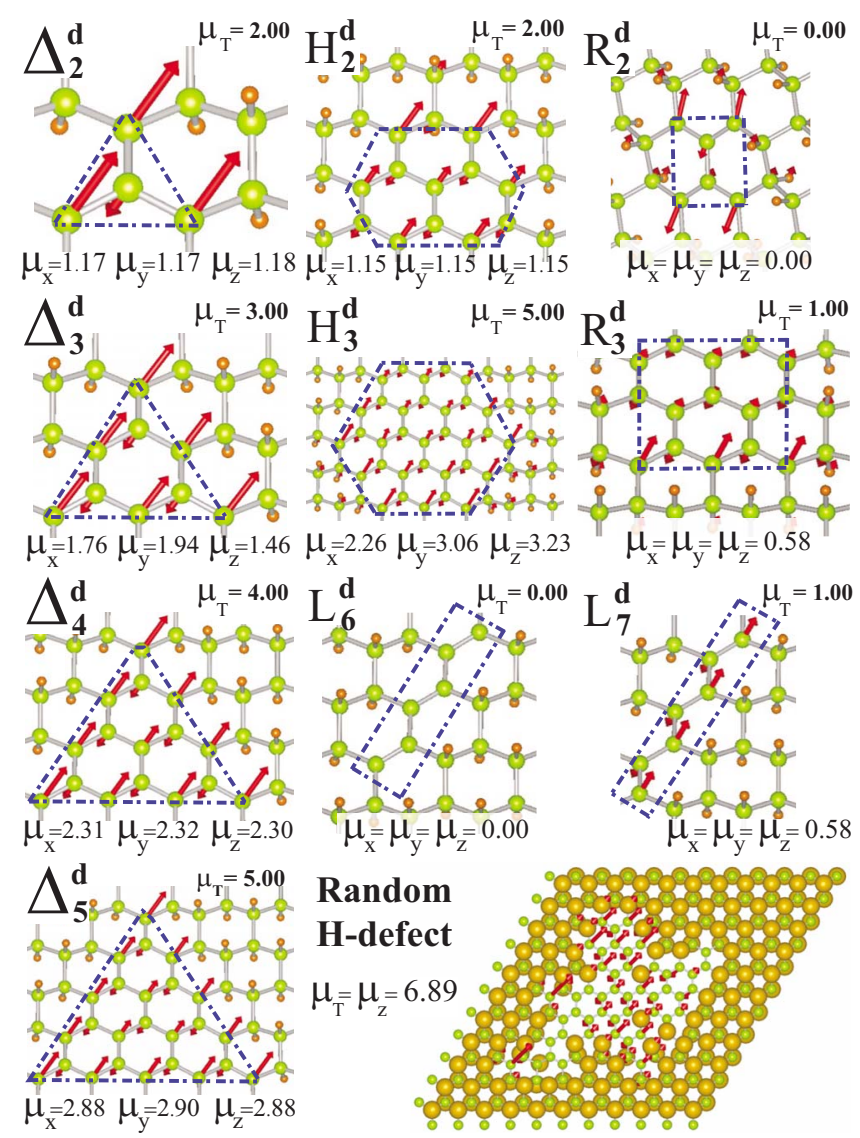

FIG. 3. (Color online) Net magnetic moments in Bohr magneton within the triangular $\Delta_{n}^{d}$, hexagonal $H_{n}^{d}$, rectangular $R_{n}^{d}$, and lane $L_{n}^{d}$ domains, which are delineated by dashed-dotted lines and have $n$ carbon atoms at their edges. Here $d$ signifies the double-sided dehydrogenation. Random shaped domain including both one and two-sided H-vacancy parts is also illustrated. 
$\pi$-bonds and hence pair their spins. At the end, $L_{4}^{s}$ has $\mu_{T}$ $=0$. For $L_{5}^{s}$ having odd number of H-vacancy, while two pairs of $\mathrm{C}$ atoms are bound by two $\pi$-bonds, $\mathrm{C}$ atom at the center has an unpaired spin and attains $\mu_{T}=1 \mu_{B}$. In a similar manner, the hexagonal domain $H_{2}^{s}$ has total of seven $\mathrm{C}$ atoms at its center and corners, all $\mathrm{H}$ atoms stripped from top side. At the bottom side, $\mathrm{H}$ atoms are relocated and hence the spins of adjacent $\mathrm{C}$ atoms are paired to result in a total net magnetic moment of $\mu=1 \mu_{B}$.

We next show in Fig. 3 that the magnetic moment of graphane can be tuned by changing the size and geometry of a given double-sided H-vacancy domain. In this case the situation is not complex and allows us to figure out the magnetic moment of the entire structure easily. Based on noncollinear calculations including the spin-orbit coupling, the direction of the unpaired spins on the $A$-type $\mathrm{C}$ atoms freed from $\mathrm{H}$ atoms is found to be opposite to that of the spins of $B$-type $\mathrm{C}$ atoms. However, instead of AFM spin ordering, lowest energy state of lane defects consisting of even number of $\mathrm{C}$ atoms is $\mathrm{NM}$ due to the entirely paired $p_{z}$ orbitals. Also, large double-sided domains including lane defects with equal number of $A$ - and $B$-type $\mathrm{C}$ atoms are found to be NM. The resulting net magnetic moment of a double-sided H-vacancy domains can be given by $\mu_{T}=\left(N_{t}-N_{b}\right) \mu_{B}$, where $N_{t}$ and $N_{b}$ denote the number of stripped $\mathrm{H}$ atoms from the top and bottom sides, respectively. Accordingly, the net magnetic moment induced in $\Delta_{2}^{d}, \Delta_{3}^{d}, \Delta_{4}^{d}$, and $\Delta_{5}^{d}$ domains are $2,3,4$, and 5 the $\mu_{B}$, respectively. The same argument can be applied to rectangular $R_{n}^{d}$, hexagonal $H_{n}^{d}$, and lane $L_{n}^{d}$ domains. Even the magnetic moment of a domain having arbitrary shape including various single-sided and double-sided $\mathrm{H}$-vacancy parts, as indicated in Fig. 3, can be retrieved by the arguments discussed above. Noninteger value of $\mu_{T}$ is due to severe distortion of structure. We also note that our results regarding to the unpaired spin of a domain and their net magnetic moment are in compliance with Lieb's theorem, ${ }^{23}$ which distinguishes $A$ - and $B$-sublattices in honeycomb structure.

In conclusion, we showed that the interaction between unpaired spins associated with $\mathrm{H}$ vacancies in graphane gives rise to interesting magnetic structures. We revealed simple physical mechanisms underlying the magnetism of single-sided and double-sided vacancy domains. For singlesided domains, owing to the tendency to pair the spins of $\pi$-orbitals of adjacent $\mathrm{C}$ atoms, some of the adsorbed $\mathrm{H}$ atoms at the bottom side are relocated. At the end, the net magnetic moments can be attained in vacancy domains depending on their size and shape. For double-sided domains, interactions underlying the generation of net magnetic moment are relatively straightforward and are in good agreement with Lieb's theorem. Since the exchange coupling between different domains are hindered by domain walls, very dense data storage can be achieved through uniform coverage of identical domains. It is also noted that a graphane flake comprising a domain with large magnetic moment can be utilized as a nontoxic marker for imaging purposes. While magnetic 2D systems attract a great deal of attention due to their tunable properties at nanoscale, our results suggest that the size and ordering of magnetic moments of hydrogen vacancy domains with thin walls can be used for future data storage and spintronics applications.

Computing resources used in this work were partly provided by the National Center for High Performance Computing of Turkey (UYBHM) under Grant No. 2-024-2007.

${ }^{1}$ K. S. Novoselov, A. K. Geim, S. V. Morozov, D. Jiang, Y. Zhang, S. V. Dubonos, I. V. Grigorieva, and A. A. Firsov, Science 306, 666 (2004).

${ }^{2}$ K. S. Novoselov, A. K. Geim, S. V. Morozov, D. Jiang, M. I. Katsnelson, I. V. Grigorieva, S. V. Dubonos, and A. A. Firsov, Nature (London) 438 197 (2005).

${ }^{3}$ Y. Zhang, Y. Tan, H. L. Stormer, and P. Kim, Nature (London) 438, 201 (2005).

${ }^{4}$ N. M. R. Peres, F. Guinea, and A. H. C. Neto, Phys. Rev. B 73, 195411 (2006)

${ }^{5}$ J. M. Pereira, Jr., P. Vasilopoulos, and F. M. Peeters, Nano Lett. 7, 946 (2007).

${ }^{6}$ X. Li, X. Wang, L. Zhang, S. Lee, and H. Dai, Science 319, 1229 (2008).

${ }^{7}$ H. Sevinçli, M. Topsakal, E. Durgun, and S. Ciraci, Phys. Rev. B 77, 195434 (2008).

${ }^{8}$ H. Şahin and R. T. Senger, Phys. Rev. B 78, 205423 (2008).

${ }^{9}$ C. Ataca, E. Aktürk, and S. Ciraci, Phys. Rev. B 79, 041406(R) (2009); C. Ataca, E. Aktürk, S. Ciraci, and H. Üstünel, Appl. Phys. Lett. 93, 043123 (2008).

${ }^{10}$ E. Durgun, S. Ciraci, W. Zhou, and T. Yildirim, Phys. Rev. Lett. 97, 226102 (2006).

${ }^{11}$ D. C. Elias, R. R. Nair, T. M. G. Mohiuddin, S. V. Morozov, P. Blake, M P. Halsall, A. C. Ferrari, D. W. Boukhvalov, M. I. Katsnelson, A. K. Geim, and K. S. Novoselov, Science 323, 610 (2009).

${ }^{12}$ J. O. Sofo, A. S. Chaudhari, and G. D. Barber, Phys. Rev. B 75, 153401 (2007).

${ }^{13}$ D. V. Boukhvalov, M. I. Katsnelson, and A. I. Lichtenstein, Phys. Rev. B 77, 035427 (2008).

${ }^{14}$ G. Kresse and J. Hafner, Phys. Rev. B 47, 558 (1993); G. Kresse and J. Furthmüller, ibid. 54, 11169 (1996).

${ }^{15}$ M. Shishkin and G. Kresse, Phys. Rev. B 74, 035101 (2006).

${ }^{16}$ H. Şahin, S. Cahangirov, M. Topsakal, E. Bekaroglu, E. Aktürk, R. T. Senger, and S. Ciraci, Phys. Rev. B 80, 155453 (2009).

${ }^{17}$ D. Hobbs, G. Kresse, and J. Hafner, Phys. Rev. B 62, 11556 (2000).

${ }^{18}$ Z. Liu, L. C. Feldman, N. H. Tolk, Z. Zhang, and P. I. Cohen, Science 312, 1024 (2006).

${ }^{19}$ L. Breaux, B. Anthony, T. Hsu, S. Banerjee, and A. Tasch, Appl. Phys. Lett. 55, 1885 (1989)

${ }^{20}$ S. Vajda, M. J. Pellin, J. P. Greeley, C. L. Marshall, L. A. Curtiss, G. A. Ballentine, J. W. Elam, S. Catillon-Mucherie, P. C. Redfern, F. Mehmood, and P. Zapol, Nature Mater. 8, 213 (2009).

${ }^{21}$ E. Durgun, R. T. Senger, H. Mehrez, H. Sevinçli, and S. Ciraci, J. Chem. Phys. 125, 121102 (2006).

${ }^{22}$ H. Lee, Y. Son, N. Park, S. Han, and J. Yu, Phys. Rev. B 72, 174431 (2005)

${ }^{23}$ E. H. Lieb, Phys. Rev. Lett. 62, 1201 (1989). 